\title{
Household COVID-19 risk and in-person schooling
}

\author{
Justin Lessler ${ }^{1 *}$, M. Kate Grabowski1,2, Kyra H. Grantz' ${ }^{1}$ Elena Badillo-Goicoechea ${ }^{3}$, C. Jessica E. Metcalf ${ }^{4}$, Carly \\ Lupton-Smith $^{5}$, Andrew S. Azman ${ }^{1,6}$, Elizabeth A. Stuart ${ }^{3,5,7}$
}

1Department of Epidemiology, Johns Hopkins Bloomberg School of Public Health, Baltimore, MD, USA. 'Department of Pathology, Johns Hopkins School of Medicine, Baltimore, MD, USA. ${ }^{3}$ Department of Mental Health, Johns Hopkins Bloomberg School of Public Health, Baltimore, MD, USA. ${ }^{4}$ Department of Ecology \& Evolutionary Biology, Princeton University, Princeton, NJ, USA. ${ }^{5}$ Department of Biostatistics, Johns Hopkins Bloomberg School of Public Health, Baltimore, MD, USA. Institute of Global Health, Faculty of Medicine, University of Geneva, Geneva, Switzerland. 'Department of Health Policy and Management, Johns Hopkins Bloomberg School of Public Health, Baltimore, MD, USA.

*Corresponding author. Email: justin@jhu.edu

In-person schooling has proved contentious and difficult to study throughout the SARS-CoV-2 pandemic. Data from a massive online survey in the United States indicates an increased risk of COVID-19-related outcomes among respondents living with a child attending school in-person. School-based mitigation measures are associated with significant reductions in risk, particularly daily symptoms screens, teacher masking, and closure of extra-curricular activities. A positive association between in-person schooling and COVID-19 outcomes persists at low levels of mitigation, but when seven or more mitigation measures are reported, a significant relationship is no longer observed. Among teachers, working outside the home was associated with an increase in COVID-19-related outcomes, but this association is similar to other occupations (e.g., healthcare, office work). While in-person schooling is associated with household COVID19 risk, this risk can likely be controlled with properly implemented school-based mitigation measures.

The role of schools in transmission, and the value of school closure, has been one of the most contentious issues of the COVID-19 pandemic. There is ongoing debate about exactly how much SARS-CoV-2 risk is posed to individuals and communities by in-person schooling. While there is general consensus that it should be possible to open schools safely with adequate mitigation measures, there is little data and even less agreement as to what level of mitigation is needed.

Many ecological studies have shown an association between in-person schooling and the speed and extent of community SARS-CoV-2 transmission (1-3), though these results have not been uniform (4). While there have been numerous outbreaks in schools and school-like settings (5-7), studies outside of outbreak settings have suggested that, when mitigation measures are in place, transmission within schools is limited and infection rates mirror that of the surrounding community $(8,9)$.

However, the ways in which in-person schooling influences community SARS-CoV-2 incidence are complex. Schools play a unique role in the social fabric of the United States and other countries, and often create potential transmission connections between otherwise disparate communities. Even if transmission in classrooms is rare, activities surrounding in-person schooling, such as student pick-up and drop-off, teacher interactions, and broader changes to behavior when school is in session could lead to increases in community transmission.

There is also a growing body of evidence that younger children (e.g., those under 10 years) are less susceptible to infection when exposed (10), though it is unclear if they are less likely to pass on the virus once infected $(11,12)$, or if this reduced susceptibility is offset by increases in number of contacts during school (13). Even when school-aged children are infected, their risk of severe disease and death is low (14). This means that one of the main reasons for a focus on schools is not the risk to students, but the risk that in-person schooling poses to teachers and family members (15), and its impact on the overall epidemic. Yet, few studies have focused on the risk in-person school poses to household members (15).

Different interpretations of the evidence and local politics have led to massive heterogeneity in approaches to schooling across the United States during the 2020-21 school year (16), running the gambit from complete cessation of in-person learning to opening completely with no mitigation measures. Most schools that have opened have made some efforts to mitigate transmission, but there is much diversity in the approaches adopted.

This hodgepodge of approaches to schooling creates a natural experiment from which we can learn about what does, and does not, work for controlling school-associated SARSCoV-2 spread. However, there is no central repository of the measures implemented across the over 130,000 schools in the United States, or health outcomes in these schools. Where data are available, they are often restricted to traditional public-school systems, though $28 \%$ of Pre-K through 12th grade students are in private or charter schools, and rarely can data be linked with individual- or household-level outcomes. 
The COVID-19 Symptom Survey provides a unique opportunity to collect and analyze data on schooling behaviors and SARS-CoV-2 related outcomes from households throughout the United States. This survey is administered through Facebook in partnership with Carnegie Mellon University and yields approximately 500,000 survey responses in the United States weekly (17). It includes questions on symptoms related to COVID-19, testing and, since late November 2020, the schooling experience of any children in the household [survey details and questionnaires are available at (18)]. Analysis weights adjust for non-response and coverage bias (see materials and methods).

We analyzed data collected over two time periods during the 2020-2021 school year (Nov. 24, 2020-Dec. 23, 2020 and Jan. 11 2021-Feb. 10, 2021). Of 2,142,887 total respondents in the 50 US states and Washington DC during this period, 576,051 (26.9\%) reported at least one child in Pre-K through high school living in their household (tables S1 and S2, Fig. 1A, and fig. S1). While larger states have more responses, the per-capita response rate was fairly consistent across states (20 per 100,000, range 10-29 per 100,000) and slightly higher in smaller states (fig. S2). Forty-nine percent $(284,789 / 576,051)$ of these respondents reported a child living in the household engaged in either full- (68.8\%) or part-time (46.0\%) in-person schooling, with substantial variation both within and between states (Fig. 1 and table S3). Overall, inperson schooling increased between the two periods from $48 \%$ to $52 \%$, though decreases were observed in some states (e.g., Arizona) (fig. S1 and table S3). Previous work has shown that household-reported rates of in-person schooling collected through the COVID-19 Symptom Survey track well with administrative data (19).

After adjusting for county-level incidence and other individual- and county-level factors (but not school-based mitigation measures; tables S1 and S2 and fig. S3), living in a household with a child engaged in full-time in-person schooling is associated with a substantial increase in the odds [adjusted odds ratio (aOR) 1.38, 95\% CI 1.30-1.47] of reporting COVID-19 like illness (CLI, fever of at least $100^{\circ} \mathrm{F}$, along with cough, shortness of breath, or difficulty breathing), loss of taste or smell (aOR 1.21, 95\% CI 1.16-1.27), or a positive SARSCoV-2 test result within the previous 14 days (aOR 1.30, 95\% CI 1.24-1.35) (Fig. 2A and table S4). Rates of reported COVID19 outcomes were positively correlated with county-level confirmed SARS-CoV-2 incidence (figs. S4 and S5). When stratifying by grade level (restricted to households reporting children in a single grade strata), we find that the strength of the associations with full-time schooling increases with grade (Fig. 2A and table S4).

The association between COVID-19 outcomes and reporting a child in the household engaged in part-time in-person schooling is attenuated but still statistically significant for
CLI (aOR 1.21, 95\% CI 1.13-1.29), loss of taste or smell (aOR $1.18,95 \%$ CI 1.13-1.24) and reporting a positive test (aOR 1.09, 95\% CI, 1.03-1.14). Among those reporting part-time schooling, the association between grade and COVID-19-related outcomes is less clear (Fig. 2A and table S4).

Respondents were asked to select all mitigation measures in place for any household child engaged in in-person schooling from a list of 14 measures (see materials and methods for wording). For students engaged in any form of in-person learning, the most common mitigation measure reported was student mask mandates ( $88 \%$, unweighted), followed by teacher mask mandates (80\%), restricted entry (e.g., no parents or caregivers allowed into school) (66\%) and extra space between desks (63\%) (see table S5 for survey weighted rates). The distribution of mitigation measures reported was similar between those reporting full- and part-time in-person schooling, though most measures were slightly more likely to be reported in the part-time setting (Fig. 2B). Besides staying with the same teacher and staying with the same students throughout the day, we found minimal evidence of clustering of mitigation measures in principal components (table S6) or hierarchical clustering analyses (fig. S6). Student mask mandates were the only intervention reported alone.

Overall, respondents reporting a household child engaged in in-person school reported a mean of 6.7 (IQR 4-9) mitigation measures in place at any school attended. Those reporting only children in part-time schooling reported more mitigation measures (mean 7.0, IQR 5-10) than those reporting only children in full-time schooling (mean 6.4, IQR 4-9). There is substantial geographic heterogeneity in the number of mitigation measures reported (Fig. 1D, fig. S7, and tables S5 and S7), with households in South Dakota reporting the least (mean 4.6, IQR 2-7), and households in Vermont reporting the most (mean 8.9, IQR 8-11).

We find a dose-response relationship with the number of mitigation measures implemented and the risk of COVID-19 outcomes among adult household members responding to the survey after adjustment for individual- and county-level factors. On average, each measure implemented is associated with a $9 \%$ decrease in the odds of CLI (aOR $0.91,95 \%$ CI 0.89 0.92 ), an $8 \%$ decrease in the odds of loss of taste or smell (aOR $0.92,95 \%$ CI $0.91-0.93$ ) and a $7 \%$ decrease in the odds of a recent positive SARS-CoV-2 test (aOR 0.93, 95\% CI 0.92-0.94) (table S8). Regression treating each individual mitigation measure as having an independent effect shows that daily symptom screening is clearly associated with greater risk reductions than the average measure (Fig. 3 and table S9), with some evidence that teacher mask mandates and cancelling extra-curricular activities are also associated with larger reductions than average. In contrast, closing cafeterias, playgrounds and use of desk shields are associated with lower risk reductions (or even risk increases); however this may reflect 
saturation effects as these are typically reported along with a high number of other measures. Notably, part-time in-person schooling is not associated with a decrease in the risk of COVID-19-related outcomes compared to full-time in-person schooling after accounting for other mitigation measures. Despite this heterogeneity in impact, we find that models including only the number of mitigation measures well approximate those where measures are modeled individually (fig. S8).

To explore what, if any, levels of mitigation are associated with elimination of the risk posed by in-person schooling, we conducted analyses where the in-person exposure groups were specific to whether $0,1-3,4-6,7-9$ or 10 or more mitigation measures were reported (Fig. 4, fig. S9, and tables S10 and S11). We found that when 7 or more mitigation measures were in place the positive association between in-person schooling and COVID-19 outcomes disappeared. This result was robust to adjustment for the expected number of interventions (i.e., generalized propensity scores) based on geographic or individual level covariates, but was less clear when propensity scores were based on both (fig. S10). Among those reporting 7 or more mitigation measures, over $80 \%$ reported student and teacher mask mandates, restricted entry, extra space between desks and no supply sharing, and over 50\% reported student cohorting, reduced class size and daily symptom screening.

The results presented here show a clear association between in-person schooling and the risk of COVID-19-related outcomes in adult household members, and that this association disappears when more than seven school-based mitigation measures are reported. However, this association may not be causal, particularly given that in-person schooling and mitigation measures are not distributed randomly in the population (Fig. 1 and tables S1 to S3, S5, S7, S10, and S11). For instance, households with a student attending in-person school tend to be in counties that are a higher percentage white (fig. S2), and contain respondents who are more likely to have recently eaten out or gone to a bar (table S2). Despite our best efforts to adjust for local incidence, individual behavior and other potential confounders, it is possible that unmeasured factors drive the observed associations; and some sub-analyses raise the possibility that complex interactions between geography and individual factors (but neither alone) may explain some of the observed results (fig. S10), though over-adjustment is a concern in these models.

To address the possibility that the association with in-person schooling could be the result of differences between urban, suburban and rural counties, local patterns of incidence, or other differences between those more and less likely to send children to school in-person we performed several stratified analyses (Fig. 5). When stratifying by propensity for inperson schooling and counties classified by size and metro status, or incidence, we found few systematic or statistically significant deviations from overall estimates, even if overall rates of outcomes differed (i.e., little evidence of effect modification by strata). We find similar results when stratifying counties by reported schooling behaviors, state, percent white, poverty and access to broadband internet (figs. S11 to S14 and table S12). The notable exception is an apparent increase in the risk associated with in-person schooling in households with a higher propensity to have children attending in-person classes (Fig. 5C).

While we were not able to specifically examine the relationship between in-person schooling, mitigation measures and risk to teachers, we were able to assess the risk associated with reporting paid work outside the home among pre-K through high school teachers. Teachers working outside the home were more likely to report COVID-19-related outcomes than those working at home (e.g., Test positive aOR 1.8, 95\% CI 1.5-2.2; fig. S15 and table S13). The confidence interval summarizing the elevation of risk overlapped with corresponding intervals associated with working in healthcare (aOR 1.7, 95\% CI 1.5-1.9) and office work (aOR 1.6, 95\% CI 1.51.7).

The results presented here provide evidence that in-person schooling poses a risk to those living in the households of students, but that this risk can be managed through commonly implemented school-based mitigation measures. This is consistent with findings from Sweden, where authors found risk to parents and teachers using a quasi-experimental approach (15). However, much remains unknown. We were unable to measure the risk posed by in-person schooling to the students themselves, nor were we able to specifically assess how different policies impact teachers and other school staff. While the interplay between school policies and local incidence is complex and, possibly, multi-directional, we find substantial variation in SARS-CoV-2 incidence regardless of the mean number of mitigation measures implemented within counties (figs. S8 and S15) and observed associations persist across study periods (figs. S17 to S19). This study also provides limited insight into the mechanisms by which in-person schooling increases risk, and it remains possible that classroom transmission plays a minor role, and other school-related activities drive risk.

This study has limitations. Measures of association between COVID-19 outcomes and key exposures may be biased if confounding factors were not fully accounted for. Though we adjust for several county-level measures of socioeconomic status, these data were not available at the individual level and are known to be associated with COVID-19 risk and attitudes about in-person schooling. Analyses stratified on urbanization, background COVID-19 risk, and propensity for inperson schooling (table S5) did not reveal substantial sensitivity to the levels of factors investigated, nor did examining 
alternative measures of individual and household COVID-19 occurrence (figs. S20 to S22), alleviating some of these concerns. Still, more formal studies that span schools with multiple policies and approaches would enhance insights into these questions.

Additionally, cross-sectional internet-based surveys have limitations and are subject to response biases. Although results are qualitatively consistent across COVID-19 outcomes [symptoms-based, test-based, and among those tested (figs. S20 to S22)], self-report has numerous limitations, for instance, we cannot robustly assess asymptomatic spread. We were also unable to evaluate compliance with or investment in reported mitigation measures, and there is potential for mitigation measures to be reported inaccurately on the survey. Survey respondents may not be representative of the full U.S. population, and while survey weights help account for non-response and coverage biases, weights calculated based on the Facebook user base were adjusted for representativeness of the wider population based only on age and gender, hence may not ensure representativeness across all covariates. However, the sample size of the survey and consistency of our findings across sub-analyses allay some of these concerns, as does assessment of non-COVID outcomes (figs. S23 and S24). Further, any response biases would have to be differential based on schooling status to bias our results away from the null.

The debate around in-person schooling in the United States has been intense, and has exacerbated differences in approach between independent school systems and individual families nationally. This lack of coordination has provided an opportunity to learn about the risks of in-person schooling, and the degree to which mitigation measures may reduce risk. The results presented here provide one dimension of evidence for decision makers to consider in the context of a complex policy landscape with many competing risks and priorities. While online surveys have their unique limitations, the wide reach of the COVID-19 Symptom Survey has allowed us to gather data from households engaged in heterogeneous schooling activities throughout the country in a way few other studies could. In analyzing these data, we find support for the idea that in-person schooling carries with it increased COVID-19 risk to household members; but also evidence that common, low cost, mitigation measures can reduce this risk.

\section{REFERENCES AND NOTES}

1. Y. Li, H. Campbell, D. Kulkarni, A. Harpur, M. Nundy, X. Wang, H. Nair, Usher Network for COVID-19 Evidence Reviews (UNCOVER) group, The temporal association of introducing and lifting non-pharmaceutical interventions with the time-varying reproduction number $(R)$ of SARS-CoV-2: A modelling study across 131 countries. Lancet Infect. Dis. 21, 193-202 (2021). doi:10.1016/S1473-3099(20)30785-4 Medline

2. S. Flaxman, S. Mishra, A. Gandy, H. J. T. Unwin, T. A. Mellan, H. Coupland, C.
Whittaker, H. Zhu, T. Berah, J. W. Eaton, M. Monod, Imperial College COVID-19 Response Team, A. C. Ghani, C. A. Donnelly, S. Riley, M. A. C. Vollmer, N. M. Ferguson, L. C. Okell, S. Bhatt, Estimating the effects of non-pharmaceutical interventions on COVID-19 in Europe. Nature 584, 257-261 (2020). doi:10.1038/s41586-020-2405-7 Medline

3. B. Yang, A. T. Huang, B. Garcia-Carreras, W. E. Hart, A. Staid, M. D. T. Hitchings, E. C. Lee, C. J. Howe, K. H. Grantz, A. Wesolowksi, J. C. Lemaitre, S. Rattigan, C. Moreno, B. Borgert, C. Dale, N. Quigley, A. Cummings, A. McLorg, K. LoMonaco, S. Schlossberg, D. Barron-Kraus, H. Shrock, UFCOVID Interventions Team, J. Lessler, C. D. Laird, D. A. T. Cummings, Effect of specific non-pharmaceutical intervention policies on SARS-CoV-2 transmission in the counties of the United States. medRxiv 2020.10.29.20221036 [Preprint]. 3 November 2020. https://doi.org/10.1101/2020.10.29.20221036.

4. D. N. Harris, E. Ziedan, S. Hassig, The Effects of School Reopenings on COVID-19 Hospitalizations [National Center for Research on Education Access and Choice (REACH), 2021]; www.reachcentered.org/publications/the-effects-of-schoolreopenings-on-covid-19-hospitalizations.

5. C. Stein-Zamir, N. Abramson, H. Shoob, E. Libal, M. Bitan, T. Cardash, R. Cayam, I. Miskin, A large COVID-19 outbreak in a high school 10 days after schools reopening, Israel, May 2020. Euro Surveill. 25, 2001352 (2020). doi:10.2807/1560-7917.ES.2020.25.29.2001352 Medline

6. A. S. Lopez, M. Hill, J. Antezano, D. Vilven, T. Rutner, L. Bogdanow, C. Claflin, I. T. Kracalik, V. L. Fields, A. Dunn, J. E. Tate, H. L. Kirking, T. Kiphibane, I. Risk, C. H. Tran, Transmission Dynamics of COVID-19 Outbreaks Associated with Child Care Facilities - Salt Lake City, Utah, April-July 2020. MMWR Morb. Mortal. Wkly. Rep. 69, 1319-1323 (2020). doi:10.15585/mmwr.mm6937e3 Medline

7. C. M. Szablewski, K. T. Chang, M. M. Brown, V. T. Chu, A. R. Yousaf, N. Anyalechi, P. A. Aryee, H. L. Kirking, M. Lumsden, E. Mayweather, C. J. McDaniel, R. Montierth, A. Mohammed, N. G. Schwartz, J. A. Shah, J. E. Tate, E. Dirlikov, C. Drenzek, T. M. Lanzieri, R. J. Stewart, SARS-CoV-2 Transmission and Infection Among Attendees of an Overnight Camp - Georgia, June 2020. MMWR Morb. Mortal. Wkly. Rep. 69, 1023-1025 (2020). doi:10.15585/mmwr.mm6931el Medline

8. S. A. Ismail, V. Saliba, J. Lopez Bernal, M. E. Ramsay, S. N. Ladhani, SARS-CoV-2 infection and transmission in educational settings: A prospective, cross-sectional analysis of infection clusters and outbreaks in England. Lancet Infect. Dis. 21, 344-353 (2021). doi:10.1016/S1473-3099(20)30882-3 Medline

9. A. Falk, A. Benda, P. Falk, S. Steffen, Z. Wallace, T. B. Høeg, COVID-19 Cases and Transmission in 17 K-12 Schools - Wood County, Wisconsin, August 31-November 29, 2020. MMWR Morb. Mortal. Wkly. Rep. 70, 136-140 (2021). doi:10.15585/mmwr.mm7004e3 Medline

10. R. M. Viner, O. T. Mytton, C. Bonell, G. J. Melendez-Torres, J. Ward, L. Hudson, C Waddington, J. Thomas, S. Russell, F. van der Klis, A. Koirala, S. Ladhani, J. Panovska-Griffiths, N. G. Davies, R. Booy, R. M. Eggo, Susceptibility to SARS-CoV2 Infection Among Children and Adolescents Compared With Adults: A Systematic Review and Meta-analysis. JAMA Pediatr. 175, 143-156 (2021). doi:10.1001/jamapediatrics.2020.4573 Medline

11. S. Flasche, W. J. Edmunds, The role of schools and school-aged children in SARSCoV-2 transmission. Lancet Infect. Dis. 21, 298-299 (2021). doi:10.1016/S14733099(20)30927-0 Medline

12. Q. Bi, J. Lessler, I. Eckerle, S. A. Lauer, L. Kaiser, N. Vuilleumier, D. A. T. Cummings, A. Flahault, D. Petrovic, I. Guessous, S. Stringhini, A. S. Azman, SEROCoV-POP Study Group, Household Transmission of SARS-CoV-2: Insights from a Population-based Serological Survey. medRxiv 2020.11.04.20225573 [Preprint]. 16 January 2021. https://doi.org/10.1101/2020.11.04.20225573.

13. J. Zhang, M. Litvinova, Y. Liang, Y. Wang, W. Wang, S. Zhao, Q. Wu, S. Merler, C. Viboud, A. Vespignani, M. Ajelli, H. Yu, Changes in contact patterns shape the dynamics of the COVID-19 outbreak in China. Science 368, 1481-1486 (2020). doi:10.1126/science.abb8001 Medline

14. J. F. Ludvigsson, L. Engerström, C. Nordenhäll, E. Larsson, Open Schools, Covid19, and Child and Teacher Morbidity in Sweden. N. Engl. J. Med. 384, 669-671 (2021). doi:10.1056/NEJMc2026670 Medline

15. J. Vlachos, E. Hertegård, H. B. Svaleryd, The effects of school closures on SARSCoV-2 among parents and teachers. Proc. Natl. Acad. Sci. U.S.A. 118, e2020834118 (2021). doi:10.1073/pnas.2020834118 Medline

16. Map: Where Has COVID-19 Closed Schools? Where Are They Open? (Education 
Week, 2020); www.edweek.org/leadership/map-where-are-schoolsclosed/2020/07.

17. F. Kreuter, N. Barkay, A. Bilinski, A. Bradford, S. Chiu, R. Eliat, J. Fan, T. Galili, D. Haimovich, B. Kim, S. LaRocca, Y. Li, K. Morris, S. Presser, T. Sarig, J. A. Salomon, K. Stewart, E. A. Stuart, R. Tibshirani, Partnering with a global platform to inform research and public policy making. Surv. Res. Methods 14, 159-163 (2020). doi:10.18148/srm/2020.v14i2.7761

18. Symptom Surveys, Delphi Epidata API (2020); https://cmudelphi.github.io/delphi-epidata/api/covidcast-signals/fb-survey.html.

19. C. Lupton-Smith, E. B. Goicoechea, M. Collins, J. Lessler, M. K. Grabowski, E. A. Stuart, Consistency between household and county measures of K-12 onsite schooling during the COVID-19 pandemic. arXiv:2103.13296 [stat.AP] (24 March 2021).

20. J. Lessler, M. K. Grabowski, K. H. Grantz, E. Badillo-Goicoechea, C. J. E. Metcalf, C. Lupton-Smith, A. S. Azman, E. A. Stuart, HopkinsIDD/inperson-schooling-covidsurvey: second release with updated supplement, version v1.1, Zenodo (2021); http://doi.org/10.5281/zenodo.4710757.

21. N. Barkay, C. Cobb, R. Eilat, T. Galili, D. Haimovich, S. LaRocca, K. Morris, T. Sarig, Weights and Methodology Brief for the COVID-19 Symptom Survey by University of Maryland and Carnegie Mellon University, in Partnership with Facebook. arXiv:2009.14675 [cs.SI] (25 September 2020).

22. Questions and Coding, Delphi Epidata API (2020); https://cmudelphi.github.io/delphi-epidata/symptom-survey/coding.html.

23. E. Dong, H. Du, L. Gardner, An interactive web-based dashboard to track COVID19 in real time. Lancet Infect. Dis. 20, 533-534 (2020). doi:10.1016/S14733099(20)30120-1 Medline

24. M. N. Wright, A. Ziegler, ranger: A Fast Implementation of Random Forests for High Dimensional Data in C++ andR. J. Stat. Softw. 77, 1-17 (2017). doi:10.18637/iss.v077.i01

\section{ACKNOWLEDGMENTS}

This research is partially based on survey results from Carnegie Mellon University's Delphi Group. Funding: Johns Hopkins University Discovery Award (EB, CL, EAS); Johns Hopkins University COVID-19 Modeling and Policy Hub Award (EB, CL, EAS); Department of Health and Human Services (JL, MKG). Author contributions: Conceptualization: JL, MKG, CJEM, ASA, EAS. Methodology: JL, MKG, EAS. Investigation: JL, MKG, EB, CL, KHG. Visualization: JL, MKG, KHG. Funding acquisition: JL, EAS. Project administration: JL, EAS. Supervision: JL, MKG, EAS. Writing - original draft: JL, MKG, KHG, EB, CJEM, CL, ASA, EAS. Writing - review and editing: JL, MKG, KHG, EB, CJEM, CL, ASA, EAS. Competing interests: Authors declare they have no competing interests. Data and materials availability: Data are freely available from the CMU Delphi Research Group to researchers at universities and non-profits as detailed at Getting Data Access - Delphi Epidata API (https://cmu-delphi.github.io/delphi-epidata/). All analytic code with dummy data sets is available at

https://github.com/HopkinsIDD/inperson-schooling-covid-survey (note this code will not reproduce paper tables and figures without obtaining underlying data from CMU). Analytic code is available at (20). This work is licensed under a Creative Commons Attribution 4.0 International (CC BY 4.0) license, which permits unrestricted use, distribution, and reproduction in any medium, provided the original work is properly cited. To view a copy of this license, visit https://creativecommons.org/licenses/by/4.0/. This license does not apply to figures/photos/artwork or other content included in the article that is credited to a third party; obtain authorization from the rights holder before using such material.

\section{SUPPLEMENTARY MATERIALS}

science.sciencemag.org/cgi/content/full/science.abh2939/DC1

Materials and Methods

Figs. S1 to S26

Tables S1 to S13

References (21-24)

MDAR Reproducibility Checklist

Data S1 to S3
27 February 2021; accepted 26 April 2021

Published online 29 April 2021

10.1126/science.abh2939 
A

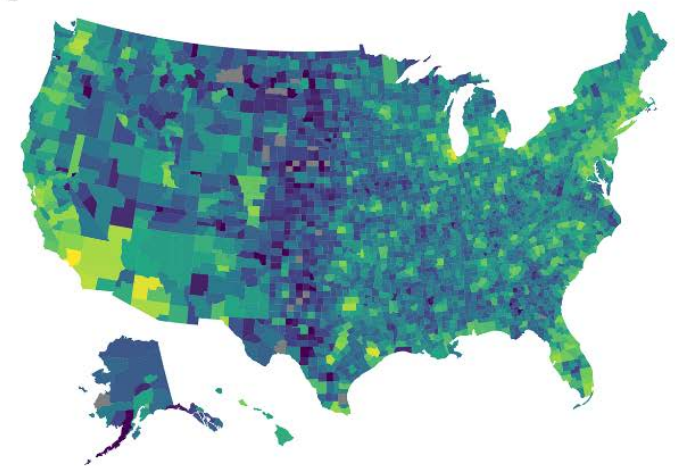

$\mathrm{N}$ respondents

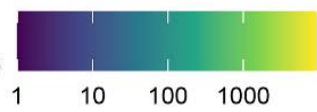

C
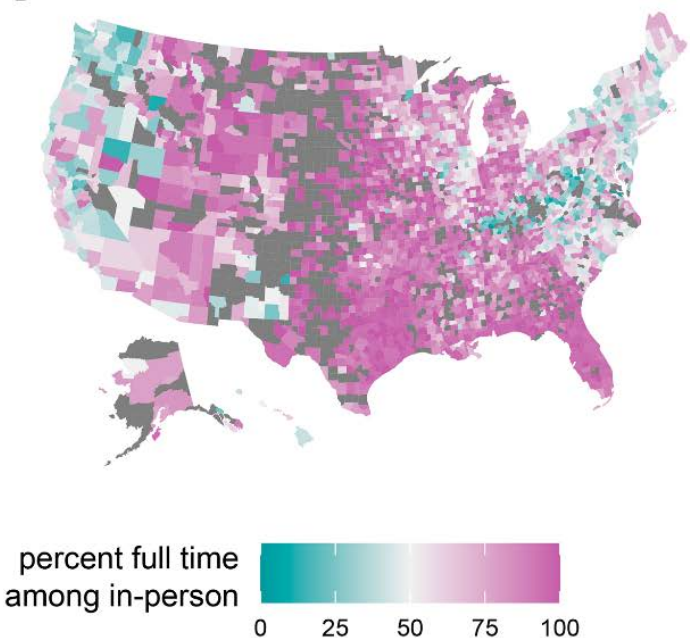

B
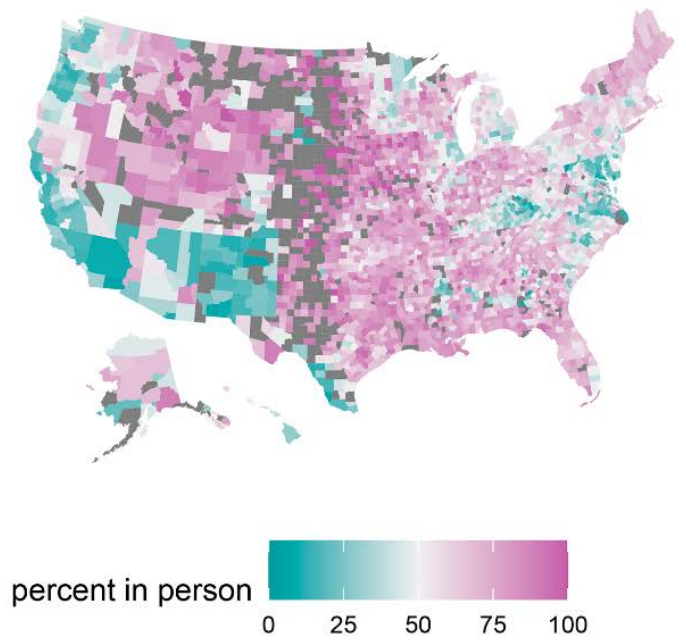

D

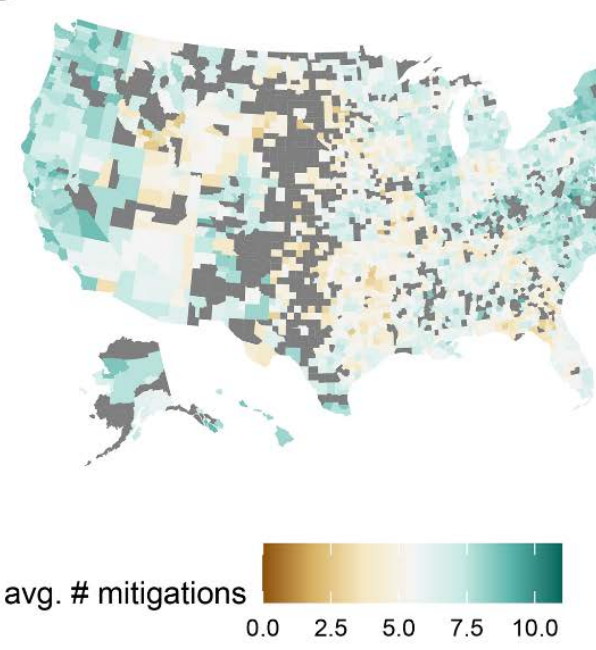

Fig. 1. Spatial distribution of survey responses. (A) Number of survey respondents reporting a school age student in the household by county. (B) Percentage of households with school age children reporting any in-person schooling by county, excluding counties with fewer than 10 responses (excluded counties in dark grey). (C) Percentage of households reporting a child in in-person schooling who report full-time in-person schooling, excluding counties with fewer than 10 reporting in-person schooling. (D) Average number of school-based mitigation measures reported for children with inperson schooling, excluding counties with fewer than 10 reporting in-person schooling. 


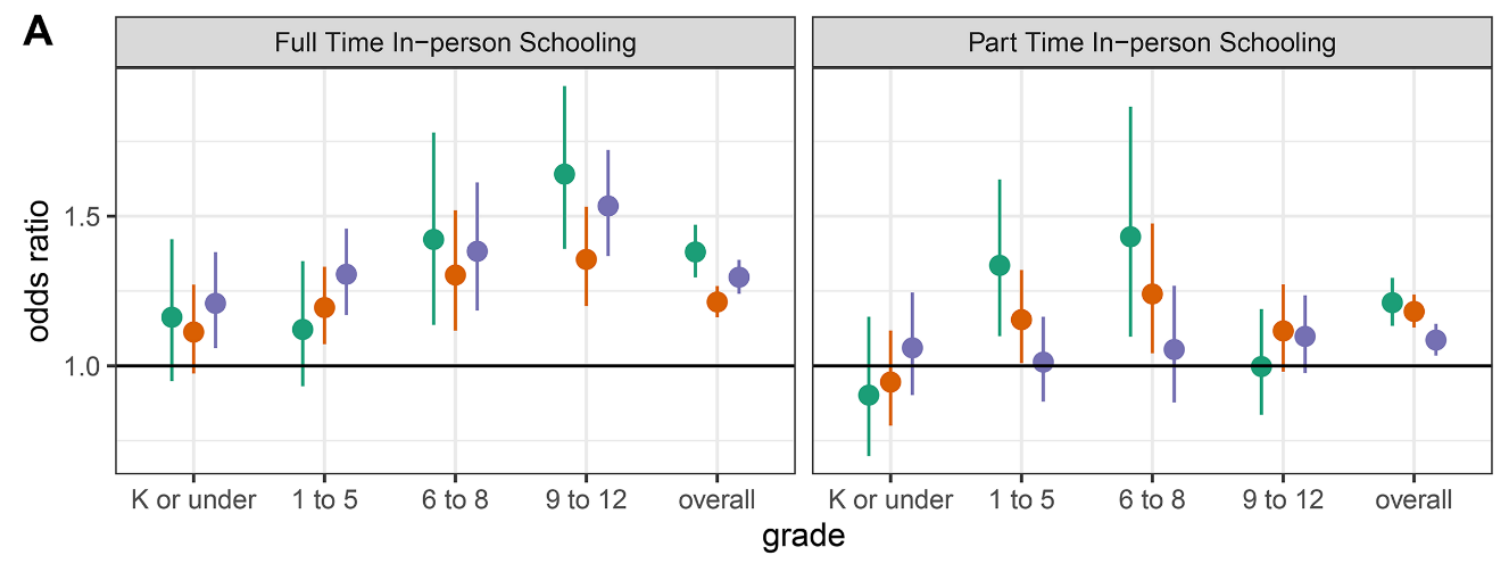

$\phi$ COVID like illness $\phi$ Loss of taste/smell $\quad$ Overall Test+

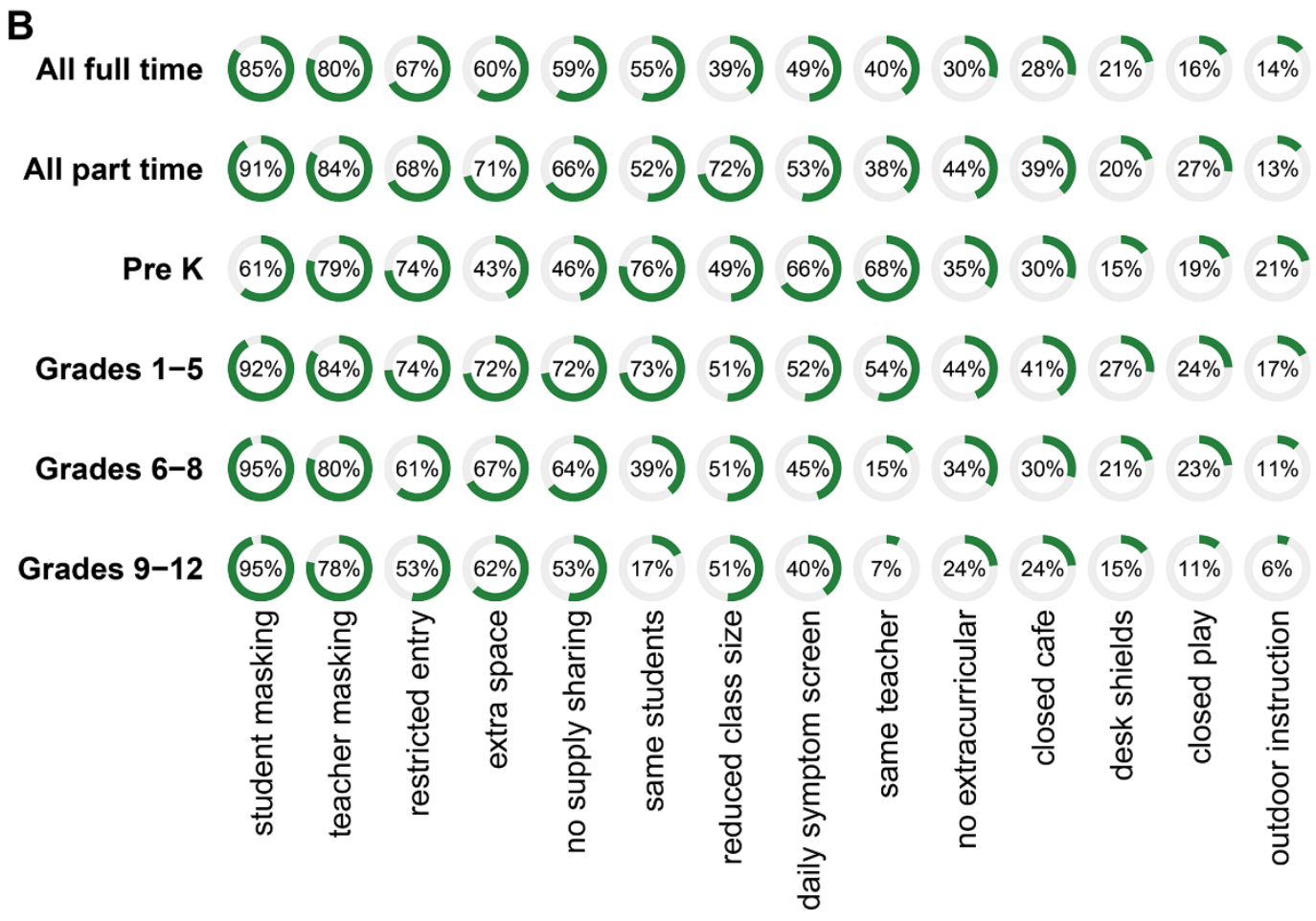

Fig. 2. Risk from in-person schooling and distribution of mitigation measures by grade. (A) Odds ratio of COVID-19-related outcomes associated with full- and part-time in-person schooling by outcome and grade level, compared to individuals with children in their household not attending in-person schooling and adjusted for individual- and county-level covariates (but not number of mitigation measures) indicating that the strength of the association increases with grade level. (B) Distribution of mitigation measures by grade level and full- versus part-time in-person status across all grades. 

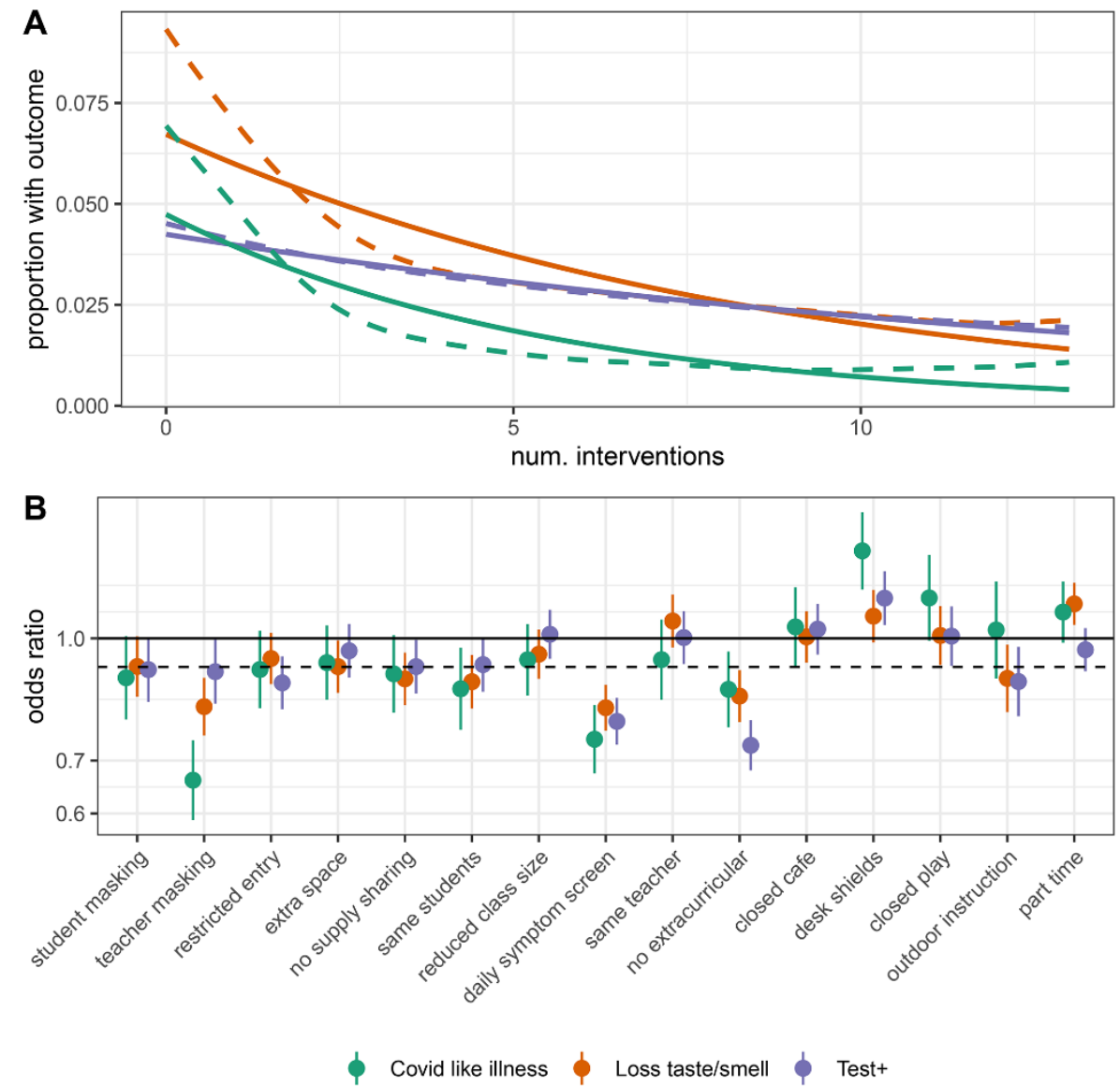

Fig. 3. Impact of individual mitigation measures. (A) Relationship between number of mitigation measures and percent reporting COVID19-related outcomes using a log-linear (solid) and spline (dashed) model. (B) Odds ratio of COVID-19-related outcomes by mitigation measure in multivariable model including all measures, versus the reduction due to a generic mitigation measure (dotted line). 
A

Covid-like illness $\quad$ Loss taste/smell $\phi$ Overall Test+
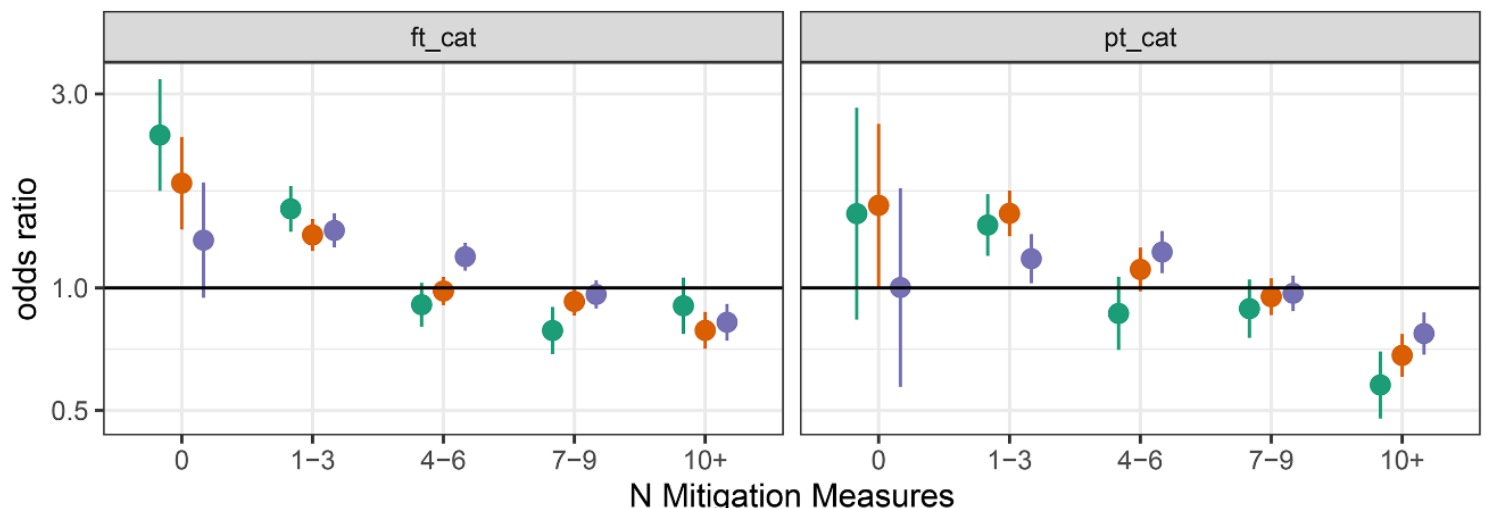

B

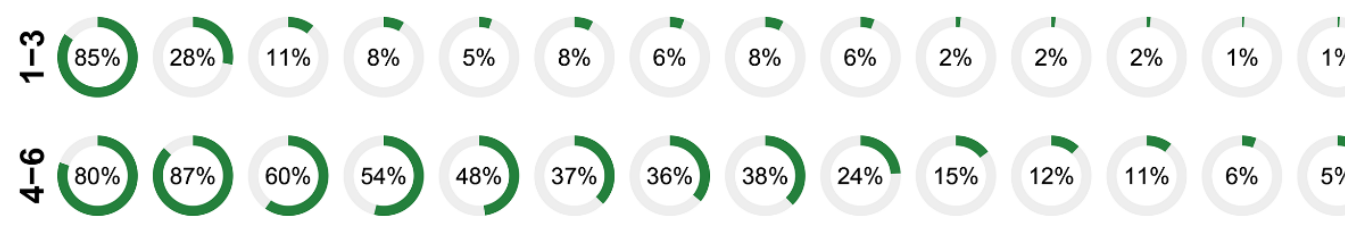

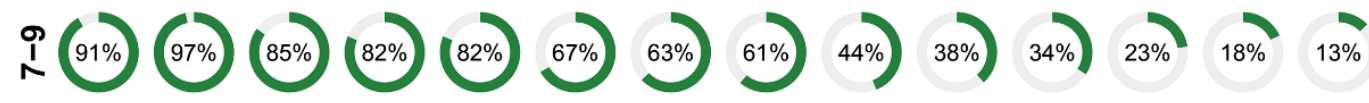

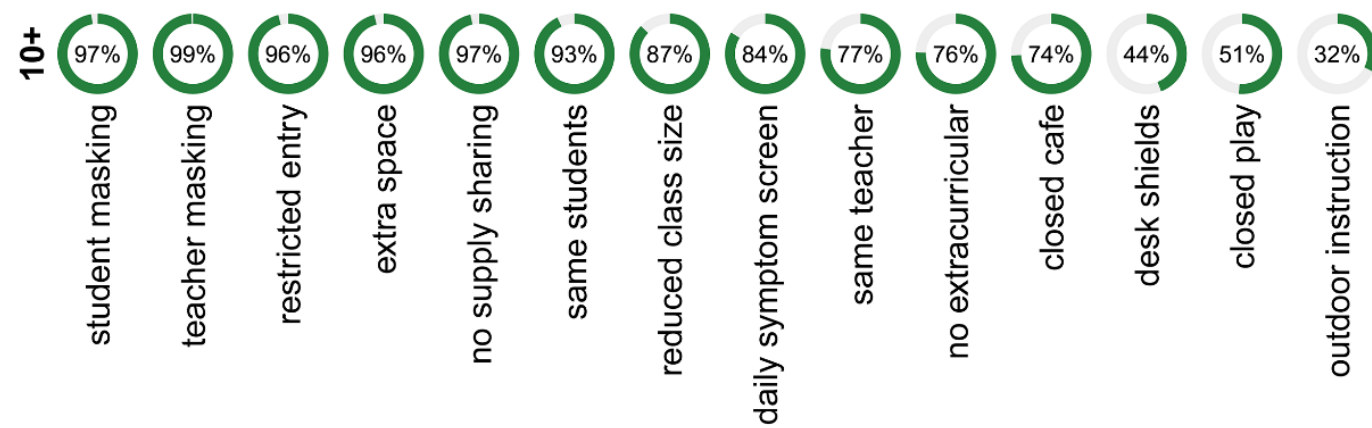

Fig. 4. Risk of in-person schooling by strata of number of reported mitigation measures. (A) Estimated risk associated with full- and part-time in-person schooling by outcome and number of mitigation measures implemented, adjusted for individual and county-level covariates. (B) Distribution of mitigation measures by total number of measures implemented. 

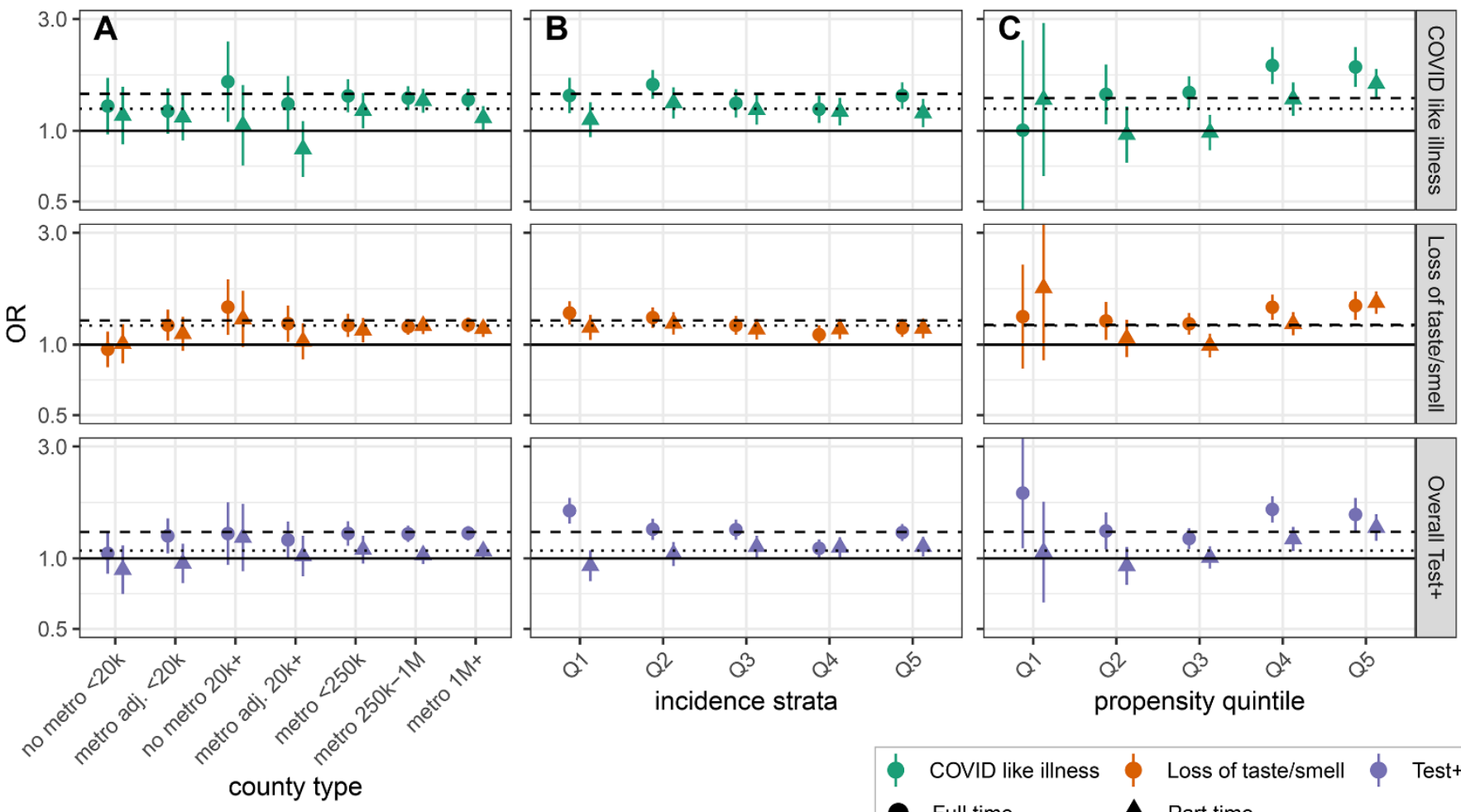

county type

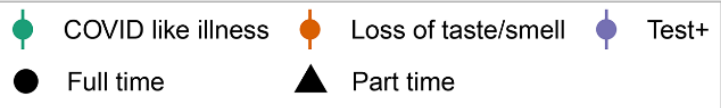

Fig. 5. Sub-group analysis of association between in-person schooling and COVID-19-related outcomes. Estimated odds ratio (versus those in strata not reporting in-person schooling) of COVID-19-related outcomes from full-time (circles, dashed lines) and part-time (triangles, dotted line) in-person schooling when data are stratified by (A) county population size and relation to metropolitan areas (metropolitan area, non-metropolitan area, adjacent to metropolitan area), (B) quintile of incidence (Q1 is lowest, Q5 is highest) and (C) propensity to report in-person schooling (Q5 most likely to have in-person schooling, Q1 least likely). Horizontal dashed and dotted lines show overall point estimates for full-time and part-time in-person instruction, respectively. 